\title{
Associations of physical activity, sedentary time, and cardiorespiratory fitness with heart rate variability in 6- to 9-year-old children: the PANIC study
}

\author{
Aapo Veijalainen ${ }^{1}$ (1) - Eero A. Haapala ${ }^{1,2}$ - Juuso Väistö ${ }^{1}$ - Marja H. Leppänen ${ }^{2,3} \cdot$ Niina Lintu $^{1} \cdot$ Tuomo Tompuri $^{1}$. \\ Santeri Seppälä ${ }^{1} \cdot$ Ulf Ekelund $^{4} \cdot$ Mika P. Tarvainen $^{5,6} \cdot$ Kate Westgate $^{7} \cdot$ Søren Brage ${ }^{7} \cdot$ Timo A. Lakka $^{1,5,8}$
}

Received: 12 May 2019 / Accepted: 10 September 2019 / Published online: 18 September 2019

(C) The Author(s) 2019

\begin{abstract}
Purpose To study the associations of physical activity (PA), sedentary time (ST), and cardiorespiratory fitness (CRF) with heart rate variability (HRV) in children.

Methods The participants were a population sample of 377 children aged 6-9 years (49\% boys). ST, light PA (LPA), moderate PA (MPA), vigorous PA (VPA), and moderate-to-vigorous PA (MVPA), and PA energy expenditure (PAEE) were assessed using a combined heart rate and movement sensor, maximal power output per kilograms of lean body mass as a measure of CRF by maximal cycle ergometer exercise test, and HRV variables (SDNN, RMSSD, LF, and HF) using 5 min resting electrocardiography. Data were analysed by linear regression adjusted for years from peak height velocity.

Results In boys, ST was inversely associated $(\beta=-0.185$ to $-0.146, p \leq 0.049)$ and MVPA, VPA, PAEE, and CRF were directly associated $(\beta=0.147$ to $0.320, p \leq 0.048)$ with HRV variables. CRF was directly associated with all HRV variables and PAEE was directly associated with RMSSD after mutual adjustment for ST, PAEE, and CRF ( $\beta=0.169$ to 0.270 , $p \leq 0.046)$. In girls, ST was inversely associated $(\beta=-0.382$ to $-0.294, p<0.001)$ and LPA, MPA, VPA, MVPA, and PAEE were directly associated with HRV variables $(\beta=0.144$ to $0.348, p \leq 0.049)$. After mutual adjustment for ST, PAEE, and CRF, only the inverse associations of ST with HRV variables remained statistically significant.

Conclusions Higher ST and lower PA and CRF were associated with poorer cardiac autonomic nervous system function in children. Lower CRF in boys and higher ST in girls were the strongest correlates of poorer cardiac autonomic function.
\end{abstract}

Keywords Physical activity $\cdot$ Cardiorespiratory fitness $\cdot$ Autonomic nervous system $\cdot$ Children

Communicated by Lori Ann Vallis.

Electronic supplementary material The online version of this article (https://doi.org/10.1007/s00421-019-04231-5) contains supplementary material, which is available to authorized users.

Aapo Veijalainen

veijalai@uef.fi

1 Institute of Biomedicine, School of Medicine, University of Eastern Finland, Kuopio Campus, PO Box 1627,

70211 Kuopio, Finland

2 Faculty of Sport and Health Sciences, University of Jyväskylä, Jyväskylä, Finland

3 Folkhälsan Research Center, Helsinki, Finland

4 Department of Sport Medicine, Norwegian School of Sport Sciences, Oslo, Norway

$\begin{array}{ll}\text { Abbreviations } \\ \text { BF\% } & \text { Body fat percentage } \\ \text { CRF } & \text { Cardiorespiratory fitness } \\ \text { CRS } & \text { Cardiometabolic risk score } \\ \text { HF } & \text { High-frequency power } \\ \text { HRV } & \text { Heart rate variability }\end{array}$

5 Department of Clinical Physiology and Nuclear Medicine, Kuopio University Hospital, Kuopio, Finland

6 Department of Applied Physics, University of Eastern Finland, Kuopio, Finland

7 MRC Epidemiology Unit, Institute of Metabolic Science, School of Clinical Medicine, University of Cambridge, Cambridge, UK

8 Kuopio Research Institute of Exercise Medicine, Kuopio, Finland 


$\begin{array}{ll}\text { LF } & \text { Low-frequency power } \\ \text { LPA } & \text { Light physical activity } \\ \text { MET } & \text { Metabolic equivalent of task } \\ \text { MPA } & \text { Moderate physical activity } \\ \text { MVPA } & \text { Moderate-to-vigorous physical activity } \\ \text { PA } & \text { Physical activity } \\ \text { PAEE } & \text { Physical activity energy expenditure } \\ \text { PANIC } & \text { Physical activity and nutrition in children } \\ \text { PHV } & \text { Peak height velocity } \\ \text { RMSSD } & \begin{array}{l}\text { Root mean square of successive RR interval } \\ \text { differences }\end{array} \\ \text { SDNN } & \text { Standard deviation of RR intervals } \\ \text { ST } & \text { Sedentary time } \\ \text { VPA } & \text { Vigorous physical activity }\end{array}$

\section{Introduction}

Globally, cardiovascular diseases cause remarkable individual, public health, and economic burden (Leal et al. 2006; Bloom et al. 2011; Laslett et al. 2012). Although cardiovascular diseases are typically affecting adults, these diseases have their origin in childhood (Celermajer and Ayer 2006). Therefore, the prevention of cardiovascular diseases, including the promotion of a physically active lifestyle, should start in childhood (Kavey et al. 2003; Celermajer and Ayer 2006).

The beneficial effects of physical activity (PA) on risk factors for cardiovascular diseases and their clinical manifestations in adults have been extensively documented (Blair and Morris 2009). Recent evidence suggests that increased PA and decreased sedentary time (ST) improve traditional cardiometabolic risk factors, such as insulin resistance and dyslipidemia, already among children (Väistö et al. 2019). However, the reduced incidence of cardiovascular diseases is only partly attributed to reduced levels of traditional risk factors due to increased PA and the mechanisms through which PA reduces cardiovascular risk are not completely understood (Green et al. 2008).

One of the mechanisms for the association between increased PA and reduced risk of cardiovascular diseases may be alterations in cardiac autonomic nervous system function, which can be assessed by heart rate variability (HRV) (Joyner and Green 2009). Decreased HRV has been associated with multiple cardiovascular diseases, risk factors, mortality and other clinical conditions in adults, but the studies in children are still limited (Villareal et al. 2002; Seppälä et al. 2014). A recent systematic literature review concluded that higher levels of moderate-to-vigorous PA (MVPA) were associated with better cardiac autonomic nervous system function in children and adolescents (Oliveira et al. 2017). However, the literature review also highlighted that the evidence on the association between $\mathrm{PA}$ and cardiac autonomic nervous system function is still limited and called for further studies investigating the associations of the whole spectrum of PA intensity and the confounding factors and physiological mechanisms for the association, such as age, sex, biological maturation, and body weight (Oliveira et al. 2017). Furthermore, few studies with inconsistent results have investigated the association between cardiorespiratory fitness (CRF) and HRV in children (Oliveira et al. 2017).

The purpose of our study was to investigate the independent and joint associations of different PA intensities, ST, and CRF with cardiac autonomic nervous system function, assessed by HRV, in children aged 6-9 years controlling comprehensively for possible confounding factors.

\section{Methods}

\section{Study design and study population}

The present cross-sectional analyses are based on the baseline data of the physical activity and nutrition in children (PANIC) study, which is a physical activity and dietary intervention and follow-up study in a population sample of children from the city of Kuopio, Finland. Altogether 736 children aged 6-9 years from primary schools of Kuopio were invited to participate in the baseline examination in 2007-2009. A total of 512 children (70\% of those invited) participated in the baseline examinations. Six children were excluded from the study at baseline because of physical disabilities that could hamper participation in the intervention or had limited time or motivation to participate in the study. The participants did not differ in sex distribution, age, or body mass index standard deviation score from all children who started the first grade in Kuopio in 2007-2009 based on data from the standard school health examinations performed for all Finnish children before the first grade (data not shown). In the present analyses, valid data for HRV, PA, ST, and CRF were available for 377 children. The study protocol was approved by the Research Ethics Committee of the Hospital District of Northern Savo, Kuopio, Finland. All participating children and their parents provided written informed consent.

\section{Study protocol}

The children and their parents or caregivers arrived at the exercise and health laboratory at the Institute of Biomedicine at 08.00 am or 09.15 am after fasting for at least $12 \mathrm{~h}$. The children were pre-informed to abstain from caffeinated drinks for at least $12 \mathrm{~h}$ and avoid strenuous PA for at least $24 \mathrm{~h}$ before the visit but continue using their medications. A physician performed a clinical examination before the maximal exercise test and the HRV assessments. The 
children were also offered a breakfast and were asked to rest to standardize the conditions before the HRV assessments and the maximal exercise test that were performed about an hour after having the breakfast. The visit was rescheduled for children who had suffered from an illness or a condition that could hamper biochemical analyses performed using blood samples, cause a risk during the exercise test, or make it difficult to perform the exercise test. The measurements were performed at a temperature of $21 \pm 1{ }^{\circ} \mathrm{C}$ and at a humidity of $30 \pm 10 \%$.

\section{Assessment of heart rate variation}

The assessment of HRV has been explained in more detail elsewhere (Seppälä et al. 2014). Briefly, electrocardiography was registered for five minutes after a 10-minute rest in a supine position and before the exercise test according to the conventional 12-lead system using the Cardiosoft ${ }^{\circledR}$ V6.5 Diagnostic System (GE Healthcare Medical Systems, Freiburg, Germany) at a frequency of $500 \mathrm{~Hz}$. The electrocardiographic data were analysed using the Kubios ${ }^{\circledR} \mathrm{HRV}$ software (Kubios Ltd., Kuopio, Finland), and the details of the techniques and analysis methods employed to assess HRV have been described elsewhere (Tarvainen et al. 2014). Briefly, the R-wave peaks were first detected using an adaptive QRS detection algorithm, and the RR interval time series (time intervals between successive $R$ waves as a function of R-wave time instants) were formed. Prior to the analysis, the data were checked for potential ectopic or aberrant beats and, if necessary, such erroneous beats were corrected using interpolation methods. The HRV variables used in the analyses included the standard deviation of all RR intervals (SDNN), a marker of overall HRV, and the root mean square of successive RR interval differences (RMSSD), a marker for parasympathetic activity (Malliani et al. 1991). These HRV variables assess HRV in the time domain with a lower value indicating a lowered parasympathetic modulation. In addition, we assessed HRV in the frequency domain by calculating high-frequency power ( $\mathrm{HF} 0.15-0.40 \mathrm{~Hz}$ ), which represents the parasympathetic modulation, low-frequency power (LF $0.04-0.15 \mathrm{~Hz}$ ), which represents a mixture of the sympathetic and parasympathetic modulation, and LF/HF, which estimates the balance between sympathetic and parasympathetic nervous system activity (Shaffer and Ginsberg 2017). For comparison purposes, we also calculated HRV variables adjusted for the mean of RR interval, as suggested elsewhere (Gąsior et al. 2018).

\section{Assessment of cardiorespiratory fitness}

CRF was assessed by a maximal exercise test using the Ergoselect $200 \mathrm{~K} \circledR$ electromagnetic bicycle ergometer with a paediatric saddle module (Ergoline, Bitz, Germany), as explained in more detail previously (Lintu et al. 2013; Tompuri et al. 2015a). The exercise test protocol included a 3 min warm-up period at $5 \mathrm{~W}$, a 1 min steady-state period at $20 \mathrm{~W}$, an exercise period with a workload increase of $1 \mathrm{~W}$ every $6 \mathrm{~s}$ until exhaustion, and a 4 min cooling-down period at $5 \mathrm{~W}$. The children were asked to keep the cadence stable and within 70-80 revolutions per minute. Exhaustion was defined as the inability to maintain the cadence above 65 revolutions per minute regardless of vigorous verbal exhortation. The exercise test was considered maximal if the reason for terminating the test indicated maximal effort and maximal cardiorespiratory capacity and if the child could not maintain the cadence above 65 revolutions per minute regardless of vigorous verbal exhortation and reached at least $90 \%$ of maximal predicted heart rate (Karila et al. 2001; Tompuri et al. 2015a). Maximal workload expressed in watts per kilograms of lean body mass was used as a measure of CRF.

\section{Assessment of physical activity and sedentary time}

PA and ST were assessed using a combined heart rate and movement sensor Actiheart ${ }^{\circledR}$ (CamNtech Ltd., Papworth, UK) for a minimum of four consecutive days without interruption, including two weekdays and two weekend days, analysed in $60 \mathrm{~s}$ epochs (Brage et al. 2005). The combined heart rate and movement sensor were attached to the child's chest with two standard electrocardiographic electrodes (Bio Protech Inc., Wonju, South Korea). The children were asked to wear the monitor continuously including sleep and waterbased activities and not to change their usual behaviour during the monitoring period. Heart rate data were cleaned (Stegle et al. 2008) and individually calibrated using parameters obtained from the maximal cycle exercise test (Brage et al. 2007) and were combined with movement sensor data to derive PA energy expenditure (PAEE). Instantaneous PAEE, i.e. PA intensity, was estimated using branched equation modelling (Brage et al. 2004) and was expressed as time spent at intensity levels of standard metabolic equivalents (METs), one MET corresponding to $71.2 \mathrm{~J} / \mathrm{min} / \mathrm{kg}$, in minutes per day. Non-wear time was taken into account during summation to minimise potential diurnal bias caused by imbalances in wear time. For these analyses, time spent in intensity categories was summarized as sedentary time (ST) ( $\leq 1.50$ METs), light PA (LPA 1.51-4.00 METs), moderate PA (MPA 4.01-7.00 METs, and vigorous PA ( $>7.00$ METs). MVPA included MPA and VPA. These broader MET thresholds have commonly been applied in studies on PA among children and youth (Janssen and Leblanc 2010). The average sleep duration, that was inferred from the combined heart rate and movement data by a trained exercise specialist and was confirmed by a physician, was subtracted from ST to obtain the final ST for the analyses (Collings 
et al. 2017). The time of falling asleep was defined as accelerometer counts decreasing to zero and heart rate to a plateau level. The time of waking up was defined as accelerometer counts increasing and remaining above zero and heart rate increasing and remaining above the plateau level. We accepted PA and ST data for the statistical analyses if there was a minimum of $48 \mathrm{~h}$ of activity recording in weekday and weekend day hours that included at least $12 \mathrm{~h}$ from morning (3-9 am), noon (9 am-3 pm), afternoon (3-9 pm), and night (9 pm-3 am) to avoid potential bias from over-representing specific times and activities of the days.

\section{Assessment of cardiometabolic risk factors}

Body weight was measured twice after overnight fasting, bladder emptied, in standing position, and in light underwear using a calibrated InBody ${ }^{\circledR} 720$ bioelectrical impedance device (Biospace, Seoul, South Korea) to accuracy of 0.1 kilograms. The mean of these two values was used in the analyses. Body height was measured three times in the Frankfurt plane without shoes by a wall-mounted stadiometer to accuracy of $0.1 \mathrm{~cm}$. The mean of two nearest values was used in the analyses. Body mass index was calculated as body weight in kilograms divided by body height in meters squared. Body mass index standard deviation score was computed by the national references (Saari et al. 2011). Overweight was defined using the age- and sex-specific cutoffs of body mass index according to the International Obesity Task Force criteria (Cole et al. 2000). Waist circumference was measured three times at the end of expiration at middistance between the bottom of the rib cage and the top of the iliac crest with a non-stretchable measuring tape to accuracy of $0.1 \mathrm{~cm}$. The mean of two nearest values was used in the analyses. Body fat percentage $(\mathrm{BF} \%)$ and lean body mass were measured bladder emptied, in supine position, in light clothing and after removing all metal objects using the Lunar Prodigy Advance ${ }^{\circledR}$ dual-energy X-ray absorptiometry device (GE Medical Systems, Madison, Wisconsin, USA) (Tompuri et al. 2015b).

A research nurse took blood samples in the morning after a $12 \mathrm{~h}$ overnight fast. Plasma glucose was measured by a hexokinase method, serum insulin by an electrochemiluminescence immunoassay, plasma triglycerides by a colorimetric enzymatic assay, and plasma high-density lipoprotein cholesterol by a homogeneous colorimetric enzymatic assay (Viitasalo et al. 2014).

A research nurse measured systolic and diastolic blood pressure from the right arm using the Heine Gamma ${ }^{\circledR}$ G7 aneroid sphygmomanometer (Heine Optotechnik, Herrsching, Germany) to accuracy of $2 \mathrm{mmHg}$. The measurement protocol included a 5 min seated resting period followed by three measurements with a 2 min interval in between. The average of all three values of systolic blood pressure and the average of all three values of diastolic blood pressure were used in the analysis.

Age-, sex-, and height-standardized $z$-scores were calculated for waist circumference, insulin, glucose, triglycerides, reciprocal of high-density lipoprotein cholesterol, and the mean of systolic and diastolic blood pressure. Thereafter, cardiometabolic risk score (CRS) was calculated as the sum of those z scores: a larger score indicating a higher cardiometabolic risk (Viitasalo et al. 2014).

\section{Assessment of maturation}

The research physician assessed pubertal status using the five-stage scale described by Tanner (Marshall and Tanner 1969, 1970). The boys were defined as having entered clinical puberty if their testicular volume assessed by an orchidometer was $\geq 4 \mathrm{~mL}$ (stage $\geq 2$ ). The girls were defined as having entered clinical puberty if their breast development had started (stage $\geq 2$ ). As almost all children were prepubertal (98\%), years from peak height velocity (PHV) were used as an indicator of maturity (Malina et al. 2015), and it was calculated separately for boys and girls using formulas provided by Moore (Moore et al. 2015).

\section{Statistical methods}

All statistical analyses were performed using the SPSS statistical software, version 24.0 (IBM Corp., Armonk, NY, USA). The analyses were performed separately for boys and girls, because we found that sex modified the associations of ST, LPA, and MPA with HRV variables. Basic characteristics were presented as arithmetic means (standard deviations, SD) or frequencies (percentages, \%) and were compared between boys and girls using the Student's $t$ test, the Mann-Whitney $U$ test, or the Chi Square test. Before the analyses, the HRV variables (SDNN, RMSSD, HF, LF, and $\mathrm{LF} / \mathrm{HF}$ ratio) were logarithmically transformed due to their skewed distributions. We used the linear regression analysis to investigate the associations of ST, LPA, MPA, VPA, MVPA, and CRF with the HRV variables. First, ST, LPA, MPA, VPA, MVPA, and CRF were forced one by one with years from PHV into the linear regression models, and then $\mathrm{BF} \%$ or CRS was additionally forced into the models. Second, ST, PAEE, and CRF were forced simultaneously into the linear regression model with years from PHV, BF\%, and CRS. The linear regression analyses were also repeated using HRV variables adjusted for the mean of RR interval $\left(\mathrm{SDNN}_{\text {adjusted }}, \mathrm{RMSSD}_{\text {adjusted }}, \mathrm{HF}_{\text {adjusted, }}, \mathrm{LF}_{\text {adjusted }}\right.$, and LF/ $\mathrm{HF}$ ratio $\left.{ }_{\text {adjusted }}\right)$. Moreover, we studied differences in the HRV variables between children with low ( $\leq$ sex-specific median) and high ( $>$ sex-specific median) PAEE and CRF and between children with low ( $\leq$ sex-specific median) 
and high (> sex-specific median) ST and CRF using general linear models adjusted for years from $\mathrm{PHV}, \mathrm{BF} \%$, and CRS. There was no considerable multicollinearity between variables used in the statistical analyses, because the Variance Inflation Factor in all analyses was under 3.4. Residuals in all models were normal. Associations with a $p$ value of $<0.05$ were considered statistically significant.

\section{Results}

\section{Basic characteristics}

Children in this study were slightly older $(7.65$ years versus 7.56 years, $p=0.042$ ) than the 512 children in the baseline study group but did not differ in height, weight, waist circumference, systolic blood pressure, or diastolic blood pressure from the 135 children who were excluded. Almost all children (98\%) were prepubertal. Boys were taller and heavier, had lower BF\%, and were further away from PHV than girls (Table 1). Boys also had a higher waist circumference, lower insulin, higher glucose, and lower triglycerides than girls (Table 1). Boys had a lower heart rate and higher mean of RR intervals than girls. Boys also accumulated less LPA and more MPA, VPA, and MVPA and had a higher PAEE and CRF than girls (Table 1).

\section{Associations of ST, PA, and CRF with HRV variables}

In boys, ST was inversely associated with SDNN, RMSSD, LF, and HF after adjustment for years from PHV (Table 2). These associations were no longer statistically significant after further adjustment for BF\% or CRS. MPA was directly associated with RMSSD after adjustment for years from PHV (Table 2). MVPA, VPA, PAEE, and CRF were directly associated with SDNN, RMSSD, LF, and HF and inversely associated with LF/HF ratio adjusted for years from PHV (Table 2). The association of MPA with RMSSD and the associations of MVPA and PAEE with LF/HF ratio were no longer statistically significant after further adjustment for $\mathrm{BF} \%$ or CRS. CRF was directly associated with SDNN, RMSSD, LF, and HF and inversely associated with LF/HF ratio after adjusted for ST, PAEE, years from PHV, BF\%, and CRS (Table 4). PAEE was directly associated with RMSSD adjusted for ST, CRF, years from PHV, BF\%, and CRS (Table 4).

In girls, ST was inversely associated with SDNN, RMSSD, LF, and HF and directly associated with LF/HF ratio after adjustment for years from PHV (Table 3). LPA, MPA, MVPA, VPA, and PAEE were directly associated with SDNN, RMSSD, LF, and HF and inversely associated with LF/HF ratio adjusted for years from PHV (Table 3). CRF was inversely associated with $\mathrm{LF} / \mathrm{HF}$ ratio after adjustment for years from PHV (Table 3). Further adjustment for BF\% or CRS had no effect on these associations, except that the inverse association with LPA and LF/HF ratio was no longer significant. Only the inverse associations of ST with SDNN, RMSSD, LF, and HF remained statistically significant after adjustment for PAEE, CRF, years from PHV, BF\%, and CRS (Table 4).

In boys, only VPA and CRF were directly associated with RMSSD $_{\text {adjusted }}$ and $\mathrm{LF}_{\text {adjusted }}$ and inversely associated with $\mathrm{LF} / \mathrm{HF}_{\text {adjusted }}$ after adjustment for years from PHV (Online resource 1). CRF was directly associated with $\mathrm{RMSSD}_{\text {adjusted }}$ and inversely with $\mathrm{LF} / \mathrm{HF}$ ratio $_{\text {adjusted }}$ after adjustments for ST, PAEE, years from PHV, BF\%, and CRS (Online resource 2).

In girls, ST was inversely associated with SDNN adjusted, RMSSD $_{\text {adjusted }}$, and HF and directly associated with LF/ HF ratio ${ }_{\text {adjusted }}$ after adjustment for years from PHV (Online resource 1). LPA was directly associated with $\mathrm{SDNN}_{\text {adjusted}}$, $\mathrm{RMSSD}_{\text {adjusted }}$, and HF after adjustment for years from PHV (Online resource 1). MPA was inversely associated with $\mathrm{LF} / \mathrm{HF}$ ratio $_{\text {adjusted }}$ after adjustment for years from PHV (Online resource 1). MVPA was directly associated with RMSSD ${ }_{\text {adjusted }}$ and $\mathrm{HF}_{\text {adjusted }}$ and inversely with LF/ HF ratio ${ }_{\text {adjusted }}$ after adjustment for years from PHV (Online resource 1). VPA and PAEE were directly associated with $\mathrm{SDNN}_{\text {adjusted }}, \mathrm{RMSSD}_{\text {adjusted }}$, and $\mathrm{LF}_{\text {adjusted }}$ and inversely associated with $\mathrm{LF} / \mathrm{HF}$ ratio $_{\text {adjusted }}$ after adjustment for years from PHV (Online resource 1). CRF was inversely associated with $\mathrm{LF} / \mathrm{HF}$ ratio $_{\text {adjusted }}$ after adjustment for years from PHV (Online resource 1). ST was inversely associated with $\mathrm{SDNN}_{\text {adjusted }}, \mathrm{RMSSD}_{\text {adjusted, }} \mathrm{LF}_{\text {adjusted }}$, and $\mathrm{HF}_{\text {adjusted }}$ after adjustments for CRF, PAEE, years from PHV, BF\%, and CRS (Online resource 2).

Boys and girls with a combination of lower CRF and lower PAEE had the lowest RMSSD and boys and girls with a combination of higher CRF and higher PAEE had the highest RMSSD adjusted for years from PHV, BF\%, and CRS ( $p$ for the main effect $=0.001$ for boys and $<0.001$ for girls, Fig. 1). These results were similar for SDNN ( $p=0.001$ for boys and $p=0.001$ for girls), LF ( $p=0.038$ for boys and $p=0.010$ for girls), and HF ( $p=0.014$ for boys and $p<0.001$ for girls) adjusted for years from PHV, BF\%, and CRS. Girls with a combination of lower CRF and lower PAEE had the highest LF/HF ratio and girls with a combination of higher CRF and higher PAEE had the lowest LF/HF ratio adjusted for years from PHV, BF\%, and CRS ( $p$ for the main effect $=0.013$ ).

Boys and girls with a combination of higher ST and lower CRF had the lowest RMSSD and boys and girls with a combination of lower ST and higher CRF had the highest RMSSD after adjustment for years from $\mathrm{PHV}, \mathrm{BF} \%$, and CRS ( $p=0.001$ for boys and $p=0.026$ for girls, Fig. 2). 
Table 1 Characteristics of children

\begin{tabular}{|c|c|c|c|c|c|}
\hline & \multicolumn{2}{|c|}{ Boys $(n=185)$} & \multicolumn{2}{|c|}{ Girls $(n=192)$} & \multirow{2}{*}{$\begin{array}{l}P \text { value } \\
\text { for sex } \\
\text { difference }\end{array}$} \\
\hline & Mean & $\mathrm{SD}$ & Mean & SD & \\
\hline Age (years) & 7.7 & 0.4 & 7.6 & 0.4 & 0.149 \\
\hline Peak height velocity (years) & -4.4 & 0.3 & -3.6 & 0.3 & $<0.001$ \\
\hline Body height $(\mathrm{cm})$ & 130 & 5.3 & 128 & 5.6 & $<0.001$ \\
\hline Body weight (kg) & 27.5 & 5.0 & 26.2 & 4.8 & 0.003 \\
\hline Body mass index standard deviation score ${ }^{\mathrm{a}}\left(\mathrm{kg} / \mathrm{m}^{2}\right)$ & -0.2 & 1.1 & -0.2 & 1.1 & 0.61 \\
\hline Body fat content $(\%)$ & 17.4 & 8.0 & 21.9 & 7.5 & $<0.001$ \\
\hline Overweight or obese $^{\mathrm{b}}(\mathrm{N}, \%)$ & $22(11.9)$ & $24(12.5)$ & 0.857 & & \\
\hline Waist circumference $(\mathrm{cm})$ & 57.5 & 5.9 & 55.6 & 5.7 & $<0.001$ \\
\hline Insulin (mU/L) & 4.2 & 2.6 & 4.7 & 2.4 & 0.008 \\
\hline Glucose (mmol/L) & 4.9 & 0.3 & 4.7 & 0.4 & $<0.001$ \\
\hline Triglycerides (mmol/L) & 0.58 & 0.25 & 0.62 & 0.25 & $\mathbf{0 . 0 3 4}$ \\
\hline High-density lipoprotein cholesterol (mmol/L) & 1.63 & 0.31 & 1.58 & 0.29 & 0.084 \\
\hline Systolic blood pressure $(\mathrm{mm} \mathrm{Hg})$ & 100.3 & 7.1 & 99.6 & 7.2 & 0.358 \\
\hline Diastolic blood pressure (mm Hg) & 62.1 & 6.8 & 61.1 & 7.5 & 0.233 \\
\hline Cardiometabolic risk score & 0.0 & 3.7 & -0.3 & 3.3 & 0.475 \\
\hline \multicolumn{6}{|l|}{ Heart rate variability variables } \\
\hline Mean heart rate (beats/min) & 83 & 10.0 & 85 & 9.7 & 0.043 \\
\hline Mean of RR intervals (ms) & 739 & 89 & 720 & 85 & $\mathbf{0 . 0 3 4}$ \\
\hline Standard deviation of all RR intervals (ms) & 62.0 & 28.8 & 58.5 & 27.9 & 0.291 \\
\hline Root mean square of successive RR interval differences (ms) & 70.2 & 43.2 & 65.5 & 39.3 & 0.537 \\
\hline Low-frequency power $\left(\mathrm{ms}^{2}\right)$ & 1309 & 985 & 1134 & 961 & 0.050 \\
\hline High-frequency power $\left(\mathrm{ms}^{2}\right)$ & 53.1 & 16.9 & 55.3 & 15.7 & 0.313 \\
\hline Ratio of low- and high-frequency power & 1.0 & 1.0 & 0.8 & 0.6 & 0.148 \\
\hline \multicolumn{6}{|l|}{ Sedentary time, physical activity, and cardiorespiratory fitness } \\
\hline Sleep time (hours/night) & 9.6 & 0.5 & 9.7 & 0.5 & 0.444 \\
\hline Sedentary time (min/day) & 231 & 131 & 242 & 130 & 0.390 \\
\hline Light physical activity (min/day) & 496 & 102 & 520 & 109 & 0.011 \\
\hline Moderate physical activity (min/day) & 103 & 56 & 82 & 47 & $<0.001$ \\
\hline Vigorous physical activity (min/day) & 30 & 26 & 16 & 16 & $<0.001$ \\
\hline Moderate-to-vigorous physical activity (min/day) & 133 & 67 & 97 & 55 & $<0.001$ \\
\hline Physical activity energy expenditure (kJ/kg/day) & 104 & 34 & 90 & 28 & $<0.001$ \\
\hline Cardiorespiratory fitness (Watt/kg) & 3.8 & 0.5 & 3.6 & 0.5 & $<0.001$ \\
\hline
\end{tabular}

$P$ values $<0.05$ indicating statistically significant differences are bolded

$S D$ standard deviation

${ }^{a}$ According to Saari et al. (2011)

${ }^{\mathrm{b}}$ According to Cole et al. (2000)

${ }^{c} t$ test or Mann-Whitney $U$ test for continuous variables and Chi-square test for being overweight or obese

These results were similar for SDNN ( $p=0.034$ for boys and $p=0.002$ for girls), LF ( $p=0.003$ for boys), and HF ( $p=0.009$ for boys and $p=0.019$ for girls) adjusted for years from $\mathrm{PHV}, \mathrm{BF} \%$, and $\mathrm{CRS}$.

\section{Discussion}

\section{Main findings}

The main finding of our study was that higher levels of PA, lower levels of ST, and higher CRF were associated 

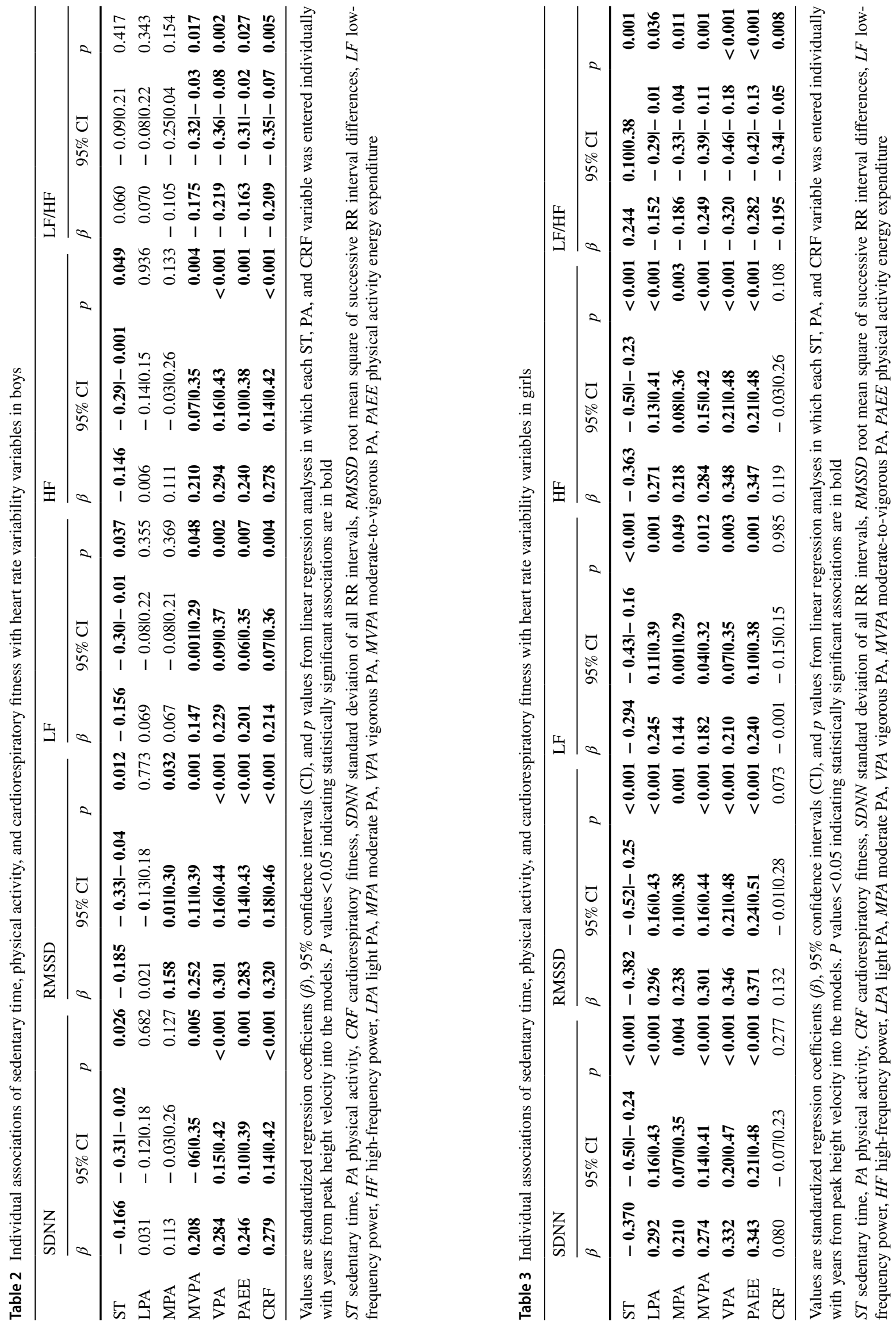


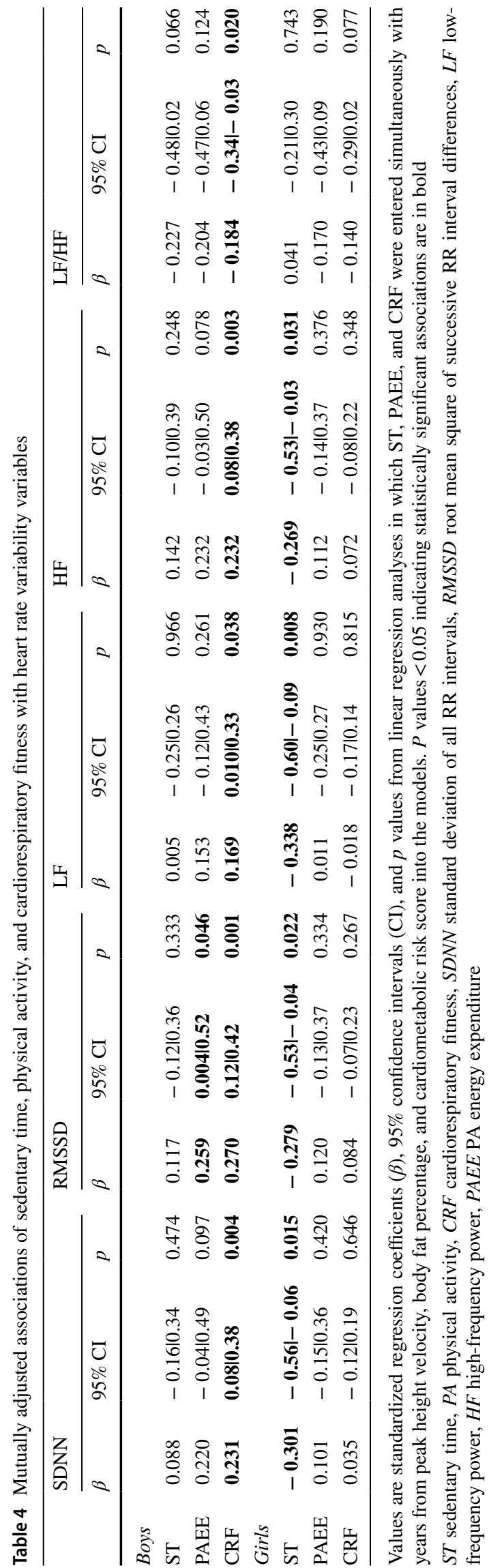

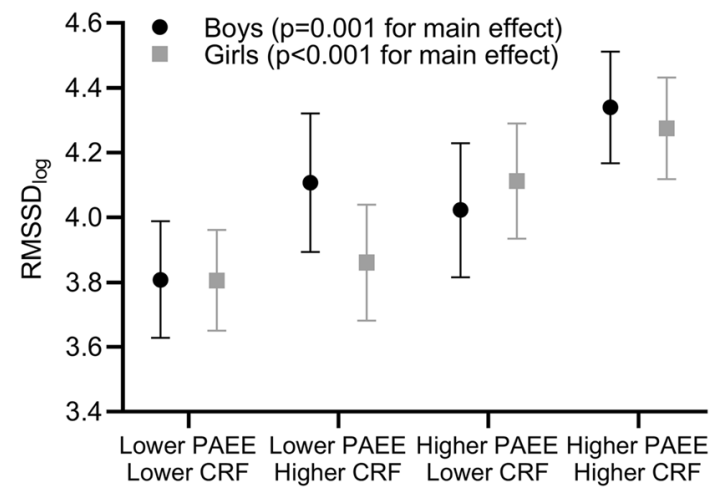

Fig. 1 Means (95\% confidence intervals) of root mean square of successive RR interval differences (RMSSD) among children with low ( $\leq$ sex-specific median) and high ( $>$ sex-specific median) maximal workload per lean body mass (CRF) and physical activity energy expenditure (PAEE)

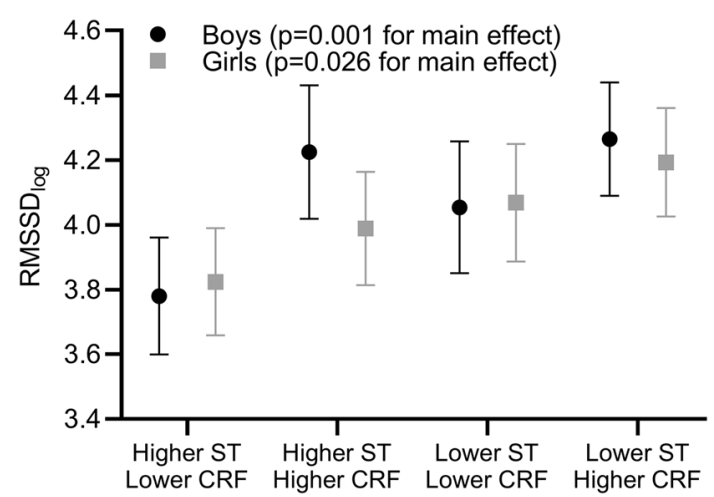

Fig. 2 Means (95\% confidence intervals) of root mean square of successive RR interval differences (RMSSD) among children with low ( $\leq$ sex-specific median) and high ( $>$ sex-specific median) maximal workload per lean body mass (CRF) and sedentary time (ST)

with better cardiac autonomic nervous system function, as indicated by higher heart rate variability, in children aged 6-9 years independent of adiposity and the cluster of cardiometabolic risk factors. CRF was the strongest determinant of cardiac autonomic nervous system function in boys and ST was the strongest determinant of it in girls. Furthermore, we found the best cardiac autonomic nervous system function in boys and girls with a combination of higher PA and higher CRF or a combination of lower ST and higher CRF and the worst cardiac autonomic nervous system function in boys and girls with a combination of lower PA and lower CRF or a combination of higher ST and lower CRF. From a clinical point of view, the results of this study suggest that the beneficial effects of an active lifestyle go beyond traditional cardiovascular risk factors. Measuring HRV could thus help to target interventions. 


\section{Associations of PA and ST with cardiac autonomic nervous system function}

In line with previous evidence (Oliveira et al. 2017), we found that more time spent in PA was associated with better cardiac autonomic nervous system function independent of adiposity and the clustering of cardiometabolic risk factors. These associations were stronger in girls than in boys. Higher levels of LPA, MPA, and VPA were related to better cardiac autonomic nervous system function in girls, whereas the positive association between PA and cardiac autonomic nervous system function in boys was mainly attributed by the strong direct association between VPA and cardiac autonomic nervous system function. However, previous studies have mainly been carried out in adolescents. Moreover, PA has usually been assessed using uniaxial accelerometer (Gutin et al. 2005; Radtke et al. 2013), which tends to underestimate PA more than combined heart rate and motion sensors (Strath et al. 2001). Furthermore, HRV variables have been shown to differ among age groups and between sexes in children that highlights the need to study the determinants of HRV in different age groups of children and in boys and girls separately (Silvetti et al. 2001).

Krishnan and coworkers showed no association of PA with HRV in children aged 9 years, but they did not fully account for the confounding effect of pubertal stages (Krishnan et al. 2009). Michels and coworkers found a positive association between PA and HRV but they did not report whether the association was statistically significant (Michels et al. 2013). Moreover, we studied the associations of not only PA but also ST and CRF with cardiac autonomic nervous system function and found that ST was a more important correlate of HRV variables than PAEE in girls and that CRF was a more important correlate of HRV variables than PAEE in boys. The results of the present study in children provide additional evidence beyond the findings of previous studies among adolescents in that the positive association between PA and cardiac autonomic nervous system function exists already in mid-childhood and is independent of adiposity and cluster of cardiometabolic risk factors and maturation in girls but may be modified by ST in girls and by CRF in boys. These cross-sectional observations are also supported by the results of an exercise intervention study showing a beneficial effect of 4-month exercise training on parasympathetic activity in obese children aged 7-11 years (Gutin et al. 2000). Exercise training had no effect on body composition and CRF was assessed using a submaximal exercise test, so no conclusion could be made on the mediating effect of CRF or adiposity on the beneficial effects of exercise training on HRV. However, longitudinal follow-up studies and especially intervention studies in children are scarce, and further studies are needed to investigate whether PA improves cardiac autonomic nervous system function in children (da Silva et al. 2014).

To our knowledge, there is only one study on the association between ST and HRV in children where there was no such association in children aged 13 years (Oliveira et al. 2017 2018). However, higher ST has been related to lower HRV in adults (Buchheit et al. 2004; Hallman et al. 2015; Miyagi et al. 2019). Consistent with these findings in adults, we observed that higher ST was associated with poorer cardiac autonomic nervous system function in children. This association was stronger in girls than in boys, and it was independent of adiposity and the cluster of cardiometabolic risk factors.

One of the explanations for the observed associations of lower PA and higher ST with lower HRV could be that lower PA and higher ST decrease blood volume and left ventricular stroke volume that results in increased heart rate due to increased sympathetic activity (Hughson and Shoemaker 2015). The regulation of hemodynamics of the human body is complex and involves neural and humoral mechanisms (Haack and Zucker 2015; Besnier et al. 2017).

\section{Association of CRF with cardiac autonomic nervous system function}

Some studies have found a positive association between CRF and cardiac autonomic nervous system function in children and adolescents, but the observations remain inconsistent (Oliveira et al. 2017, 2018). One explanation for the discrepancy may be that most of the previous studies have assessed CRF using indirect field tests or have used measures of CRF scaled by body mass (Oliveira et al. 2017) that could be confounded by adiposity and does not reflect physiological aerobic capacity (Ahn et al. 2013; Krachler et al. 2015; Tompuri et al. 2015a).

We observed that higher CRF was associated with better cardiac autonomic nervous system function independent of PA, ST, adiposity, and other cardiometabolic risk factors in children, particularly boys. In addition, CRF partly explained the positive associations of PA with HRV variables in boys suggesting that CRF mediates the relationship between PA and cardiac autonomic nervous system function.

The mechanisms underlying the association between CRF and cardiac autonomic nervous system function may be similar to those between PA and cardiac autonomic function, because especially more vigorous PA has been found to improve CRF (Ortega et al. 2008b). Although we showed a stronger association of PA with HRV in girls than in boys, the association of CRF with HRV was weaker in girls. The improvement of CRF by increasing PA has been observed to be smaller in girls than in boys that may be explained by differences in maturation and adiposity between sexes (Ortega et al. 2007, 2008b). However, 
genetic factors may be a stronger determinant of CRF than habitual PA particularly in children (Kemper and Koppes 2006; Ortega et al. 2008a; Martínez-Vizcaíno and Sánchez-López 2008). Furthermore, genetic factors also partly regulate cardiac autonomic nervous system function and may thus partly explain the association between CRF and cardiac autonomic function (Hautala et al. 2009).

\section{Joint associations of CRF, PA and ST with HRV}

We found that boys and girls with a combination of lower PAEE and lower CRF or a combination of higher ST and lower CRF had poorer cardiac autonomic nervous system function than boys and girls with a combination of higher PAEE and higher CRF or a combination of lower ST and higher CRF. Another study showed that adults with higher PA and CRF had higher SDNN and RMSSD than adults with a low PA and CRF (Buchheit and Gindre 2006). However, we observed no differences in RMSSD between boys with higher ST and higher CRF and boys with a combination of lower ST and higher CRF. This suggests that higher CRF may counteract the negative effects of higher ST with cardiac autonomic nervous system function in boys. Furthermore, we found no difference in RMSSD between girls with lower PAEE regardless of the level of CRF. However, RMSSD was higher in girls with higher PAEE and CRF than girls with lower PAEE and lower or higher CRF. These results suggest that PA more beneficially affects cardiac autonomic nervous system function than CRF in girls. The observed differences in the associations of PA, ST, and CRF with cardiac autonomic nervous system function between sexes may be partly explained by differences in maturity, adiposity, insulin, glucose, PA, and CRF between sexes.

\section{Strength and limitations}

The strengths of our study include a relatively large population sample of mainly prepubertal girls and boys with a narrow age range, the objective assessment of PA and ST with combined heart rate and movement sensing, the assessment of CRF by maximal exercise test and using a measure that is not confounded by body size or adiposity, the assessment of cardiac autonomic nervous system function using a wide range of HRV variables, and the opportunity to control for adiposity, other cardiometabolic risk factors, and maturation in the statistical analyses. However, we cannot draw any firm conclusions on causality from the observed relationships of PA, ST, and CRF with cardiac autonomic nervous system function because of the cross-sectional study design.

\section{Main conclusion}

We find higher levels of PA, lower levels of ST, and higher CRF to be associated with better cardiac autonomic function in children. These underline the importance of promoting healthy lifestyle in children. Undoubtedly the dysfunction of the autonomic nervous system plays an important role in cardiovascular health. However, the mechanisms behind that association and how it is modified with lifestyle interventions, especially in the long-term starting from childhood, remains an interesting topic for further studies.

Acknowledgements Open access funding provided by University of Eastern Finland (UEF) including Kuopio University Hospital. We are grateful to the children and their parents for participating in the PANIC study. We are also indebted to the members of the PANIC research team for their contribution in acquisition of data. We would also like to express our gratitude to Stefanie Hollidge from MRC Epidemiology Unit, University of Cambridge, for her assistance in processing the PA data. The PANIC study has financially been supported by grants from Ministry of Education and Culture of Finland, Ministry of Social Affairs and Health of Finland, Social Insurance Institution of Finland, Research Committee of the Kuopio University Hospital Catchment Area (State Research Funding), Finnish Cultural Foundation, Finnish Innovation Fund Sitra, Foundation for Paediatric Research, Juho Vainio Foundation, Diabetes Research Foundation in Finland, Finnish Foundation for Cardiovascular Research, Yrjö Jahnsson Foundation, Paavo Nurmi Foundation, and the city of Kuopio. KW and SB were supported by the UK Medical Research Council (MC_UU_12015/3) and the NIHR Cambridge Biomedical Research Centre (IS-BRC-1215-20014).

Author contributions All authors have contributed to writing the manuscript and have read and approved the final version. AV has performed the statistical analyses. AV and EAH have drafted the manuscript. AV has finalized the manuscript and EAH has done the figures.MT and SS have contributed to HRV analyses. MT, TT, and MHL have contributed to the interpretation of $\mathrm{HRV}$ data. $\mathrm{SB}, \mathrm{KW}$, and $\mathrm{UE}$ have contributed to processing and interpreting the Actiheart ${ }^{\circledR}$ data JV has been involved in processing and interpreting the Actiheart ${ }^{\circledR}$ data. TT has carried out the clinical examinations and has supervised the maximal exercise tests. NL has been involved in processing and interpreting the exercise test data. TAL has designed and executed the PANIC study and has been responsible for funding the study.

\section{Compliance with ethical standards}

Conflict of interest The authors declare that they have no conflict of interest.

Open Access This article is distributed under the terms of the Creative Commons Attribution 4.0 International License (http://creativeco mmons.org/licenses/by/4.0/), which permits unrestricted use, distribution, and reproduction in any medium, provided you give appropriate credit to the original author(s) and the source, provide a link to the Creative Commons license, and indicate if changes were made. 


\section{References}

Ahn B, McMurray R, Harrell J (2013) Scaling of VO2max and its relationship with insulin resistance in children. Pediatr Exerc Sci 25:43-51

Besnier F, Labrunée M, Pathak A et al (2017) Exercise traininginduced modification in autonomic nervous system: an update for cardiac patients. Ann Phys Rehabil Med 60:27-35. https:// doi.org/10.1016/J.REHAB.2016.07.002

Blair SN, Morris JN (2009) Healthy hearts-and the universal benefits of being physically active: physical activity and health. Ann Epidemiol 19:253-256. https://doi.org/10.1016/j.annep idem.2009.01.019

Bloom DE, Cafiero ET, Jané-Llopis E et al (2011) The global economic burden of non-communicable diseases. In: World Economic Forum, Geneva

Brage S, Brage N, Franks PW et al (2004) Branched equation modeling of simultaneous accelerometry and heart rate monitoring improves estimate of directly measured physical activity energy expenditure. J Appl Physiol 96:343-351. https://doi.org/10.1152/ japplphysiol.00703.2003

Brage S, Brage N, Franks PW et al (2005) Reliability and validity of the combined heart rate and movement sensor actiheart. Eur J Clin Nutr 59:561-570. https://doi.org/10.1038/sj.ejcn.1602118

Brage S, Ekelund U, Brage N et al (2007) Hierarchy of individual calibration levels for heart rate and accelerometry to measure physical activity. J Appl Physiol 103:682-692. https://doi.org/10.1152/ japplphysiol.00092.2006

Buchheit M, Gindre C (2006) Cardiac parasympathetic regulation: respective associations with cardiorespiratory fitness and training load. Am J Physiol Heart Circ Physiol 291:H451-H458. https ://doi.org/10.1152/ajpheart.00008.2006

Buchheit M, Simon C, Viola AU et al (2004) Heart rate variability in sportive elderly: relationship with daily physical activity. Med Sci Sports Exerc 36:601-605

Celermajer DS, Ayer JGJ (2006) Childhood risk factors for adult cardiovascular disease and primary prevention in childhood. Heart 92:1701-1706. https://doi.org/10.1136/hrt.2005.081760

Cole TJ, Bellizzi MC, Flegal KM, Dietz WH (2000) Establishing a standard definition for child overweight and obesity worldwide: international survey. BMJ 320:1240-1243

Collings PJ, Westgate K, Väistö J et al (2017) Cross-sectional associations of objectively-measured physical activity and sedentary time with body composition and cardiorespiratory fitness in midchildhood: the PANIC study. Sports Med 47:769-780. https://doi. org/10.1007/s40279-016-0606-x

da Silva CC, Pereira LM, Cardoso JR et al (2014) The effect of physical training on heart rate variability in healthy children: a systematic review with meta-analysis. Pediatr Exerc Sci 26:147-158. https ://doi.org/10.1123/pes.2013-0063

Gąsior JS, Sacha J, Pawłowski M et al (2018) Normative values for heart rate variability parameters in school-aged children: simple approach considering differences in average heart rate. Front Physiol 9:1495. https://doi.org/10.3389/fphys.2018.01495

Green DJ, O'Driscoll G, Joyner MJ, Cable NT (2008) Exercise and cardiovascular risk reduction: time to update the rationale for exercise? J Appl Physiol 105:766-768. https://doi.org/10.1152/ japplphysiol.01028.2007

Gutin B, Barbeau P, Litaker MS et al (2000) Heart rate variability in obese children: relations to total body and visceral adiposity, and changes with physical training and detraining. Obes Res 8:12-19. https://doi.org/10.1038/oby.2000.3

Gutin B, Howe C, Johnson MH et al (2005) Heart rate variability in adolescents: relations to physical activity, fitness, and adiposity. Med Sci Sports Exerc 37:1856-1863
Haack KKV, Zucker IH (2015) Central mechanisms for exercise training-induced reduction in sympatho-excitation in chronic heart failure. Auton Neurosci 188:44-50. https://doi.org/10.1016/J. AUTNEU.2014.10.015

Hallman DM, Sato T, Kristiansen J et al (2015) Prolonged sitting is associated with attenuated heart rate variability during sleep in Blue-Collar workers. Int J Environ Res Public Health 12:1481114827. https://doi.org/10.3390/ijerph121114811

Hautala AJ, Kiviniemi AM, Tulppo MP (2009) Individual responses to aerobic exercise: the role of the autonomic nervous system. Neurosci Biobehav Rev 33:107-115. https://doi.org/10.1016/j. neubiorev.2008.04.009

Hughson RL, Shoemaker JK (2015) Autonomic responses to exercise: deconditioning/inactivity. Auton Neurosci 188:32-35. https://doi.org/10.1016/J.AUTNEU.2014.10.012

Janssen I, Leblanc AG (2010) Systematic review of the health benefits of physical activity and fitness in school-aged children and youth. Int J Behav Nutr Phys Act 7:40. https://doi. org/10.1186/1479-5868-7-40

Joyner MJ, Green DJ (2009) Exercise protects the cardiovascular system: effects beyond traditional risk factors. J Physiol 587:55515558. https://doi.org/10.1113/jphysiol.2009.179432

Karila C, de Blic J, Waernessyckle S et al (2001) Cardiopulmonary exercise testing in children: an individualized protocol for workload increase. Chest 120:81-87

Kavey R-EW, Daniels SR, Lauer RM et al (2003) American Heart Association guidelines for primary prevention of atherosclerotic cardiovascular disease beginning in childhood. Circulation 107:1562-1566

Kemper HCG, Koppes LLJ (2006) Linking physical activity and aerobic fitness: are we active because we are fit, or are we fit because we are active? Pediatr Exerc Sci 18:173-181. https:// doi.org/10.1123/pes.18.2.173

Krachler B, Savonen K, Lakka T (2015) Obesity is an important source of bias in the assessment of cardiorespiratory fitness. Am Heart J. https://doi.org/10.1016/j.ahj.2015.08.016

Krishnan B, Jeffery A, Metcalf B et al (2009) Gender differences in the relationship between heart rate control and adiposity in young children: a cross-sectional study (EarlyBird 33). Pediatr Diabetes 10:127-134. https://doi.org/10.111 1/j.1399-5448.2008.00455.x

Laslett LJ, Alagona P, Clark BA et al (2012) The worldwide environment of cardiovascular disease: prevalence, diagnosis, therapy, and policy issues: a report from the American College of Cardiology. J Am Coll Cardiol 60:S1-49. https://doi.org/10.1016/j. jacc.2012.11.002

Leal J, Luengo-Fernández R, Gray A et al (2006) Economic burden of cardiovascular diseases in the enlarged European Union. Eur Heart J 27:1610-1619. https://doi.org/10.1093/eurheartj/ehi733

Lintu N, Tompuri T, Viitasalo A et al (2013) Cardiovascular fitness and haemodynamic responses to maximal cycle ergometer exercise test in children 6-8 years of age. J Sports Sci. https://doi. org/10.1080/02640414.2013.845681

Malina RM, Rogol AD, Cumming SP et al (2015) Biological maturation of youth athletes: assessment and implications. Br J Sports Med 49:852-859. https://doi.org/10.1136/bjsports-2015-094623

Malliani A, Pagani M, Lombardi F, Cerutti S (1991) Cardiovascular neural regulation explored in the frequency domain. Circulation 84:482-492

Marshall WA, Tanner JM (1969) Variations in pattern of pubertal changes in girls. Arch Dis Child 44:291-303

Marshall WA, Tanner JM (1970) Variations in the pattern of pubertal changes in boys. Arch Dis Child 45:13-23

Martínez-Vizcaíno V, Sánchez-López M (2008) Relationship between physical activity and physical fitness in children and 
adolescents. Rev Española Cardiol English Ed 61:108-111. doi: 10.1016/S1885-5857(08)600845

Michels N, Clays E, De Buyzere M et al (2013) Determinants and reference values of short-term heart rate variability in children. Eur J Appl Physiol 113:1477-1488. https://doi.org/10.1007/ s00421-012-2572-9

Miyagi R, Sasawaki Y, Shiotani H (2019) The influence of shortterm sedentary behavior on circadian rhythm of heart rate and heart rate variability. Chronobiol Int 36:374-380. https://doi. org/10.1080/07420528.2018.1550422

Moore SA, McKay HA, Macdonald H et al (2015) Enhancing a somatic maturity prediction model. Med Sci Sports Exerc 47:1755-1764. https://doi.org/10.1249/MSS.0000000000000588

Oliveira RS, Barker AR, Wilkinson KM et al (2017) Is cardiac autonomic function associated with cardiorespiratory fitness and physical activity in children and adolescents? A systematic review of cross-sectional studies. Int J Cardiol 236:113-122. https://doi. org/10.1016/j.ijcard.2017.02.022

Oliveira R, Barker A, Williams C (2018) Cardiac autonomic function, cardiovascular risk and physical activity in adolescents. Int J Sports Med 39:89-96. https://doi.org/10.1055/s-0043-118850

Ortega FB, Ruiz JR, Mesa JL et al (2007) Cardiovascular fitness in adolescents: the influence of sexual maturation status - the AVENA and EYHS studies. Am J Hum Biol 19:801-808. https://doi. org/10.1002/ajhb.20640

Ortega FB, Ruiz JR, Castillo MJ, Sjöström M (2008a) Physical fitness in childhood and adolescence: a powerful marker of health. Int $\mathbf{J}$ Obes (Lond) 32:1-11. https://doi.org/10.1038/sj.ijo.0803774

Ortega FB, Ruiz JR, Hurtig-Wennlöf A, Sjöström M (2008b) Physically active adolescents are more likely to have a healthier cardiovascular fitness level independently of their adiposity status. The European youth heart study. Rev Esp Cardiol 61:123-129

Radtke T, Kriemler S, Eser P et al (2013) Physical activity intensity and surrogate markers for cardiovascular health in adolescents. Eur J Appl Physiol 113:1213-1222. https://doi.org/10.1007/s0042 1-012-2542-2

Saari A, Sankilampi U, Hannila M-L et al (2011) New Finnish growth references for children and adolescents aged 0 to 20 years: length/ height-for-age, weight-for-length/height, and body mass indexfor-age. Ann Med 43:235-248. https://doi.org/10.3109/07853 890.2010.515603

Seppälä S, Laitinen T, Tarvainen MP et al (2014) Normal values for heart rate variability parameters in children $6-8$ years of age: the PANIC Study. Clin Physiol Funct Imaging 34:290-296. https:// doi.org/10.1111/cpf.12096
Shaffer F, Ginsberg JP (2017) An overview of heart rate variability metrics and norms. Front Public Health 5:258. https://doi. org/10.3389/fpubh.2017.00258

Silvetti MS, Drago F, Ragonese P (2001) Heart rate variability in healthy children and adolescents is partially related to age and gender. Int J Cardiol 81:169-174. https://doi.org/10.1016/S0167 -5273(01)00537-X

Stegle O, Fallert S, MacKay DJC, Brage S (2008) Gaussian process robust regression for noisy heart rate data. IEEE Trans Biomed Eng 55:2143-2151. https://doi.org/10.1109/TBME.2008.923118

Strath SJ, Bassett DR, Swartz AM, Thompson DL (2001) Simultaneous heart rate-motion sensor technique to estimate energy expenditure. Med Sci Sports Exerc 33:2118-2123

Tarvainen MP, Niskanen J-P, Lipponen JA et al (2014) Kubios HRV - heart rate variability analysis software. Comput Methods Progr Biomed 113:210-220. https://doi.org/10.1016/j. cmpb.2013.07.024

Tompuri T, Lintu N, Savonen K et al (2015a) Measures of cardiorespiratory fitness in relation to measures of body size and composition among children. Clin Physiol Funct Imaging 35:469-477. https:// doi.org/10.1111/cpf.12185

Tompuri TT, Lakka TA, Hakulinen M et al (2015b) Assessment of body composition by dual-energy X-ray absorptiometry, bioimpedance analysis and anthropometrics in children: the Physical Activity and Nutrition in Children study. Clin Physiol Funct Imaging 35:21-33. https://doi.org/10.1111/cpf.12118

Väistö J, Haapala EA, Viitasalo A et al (2019) Longitudinal associations of physical activity and sedentary time with cardiometabolic risk factors in children. Scand J Med Sci Sports 29:113-123. https ://doi.org/10.1111/sms.13315

Viitasalo A, Lakka TA, Laaksonen DE et al (2014) Validation of metabolic syndrome score by confirmatory factor analysis in children and adults and prediction of cardiometabolic outcomes in adults. Diabetologia 57:940-949. https://doi.org/10.1007/s0012 5-014-3172-5

Villareal RP, Liu BC, Massumi A (2002) Heart rate variability and cardiovascular mortality. Curr Atheroscler Rep 4:120-127

Publisher's Note Springer Nature remains neutral with regard to jurisdictional claims in published maps and institutional affiliations. 